\title{
The amino acid substitution affects cellular response to mistranslation
}

\author{
Matthew D. Berg (DD , ${ }^{1,2, *,{ }^{\dagger}}$ Yanrui Zhu, ${ }^{1, \dagger}$ Bianca Y. Ruiz, ${ }^{2}$ Raphaël Loll-Krippleber, ${ }^{3}$ Joshua Isaacson, ${ }^{4}$ Bryan-Joseph San Luis, ${ }^{5}$ \\ Julie Genereaux, ${ }^{1}$ Charles Boone (D) , Judit Villén, ${ }^{2}$ Grant W. Brown (D) , ${ }^{3}$ and Christopher J. Brandl (iD 1,* \\ ${ }^{1}$ Department of Biochemistry, The University of Western Ontario, London, ON N6A 3K7, Canada, \\ ${ }^{2}$ Department of Genome Sciences, University of Washington, Seattle, WA 98195, USA, \\ ${ }^{3}$ Department of Biochemistry, Donnelly Centre for Cellular and Biomolecular Research, University of Toronto, Toronto, ON M5S, Canada, \\ ${ }^{4}$ Department of Biology, The University of Western Ontario, London, ON N6A 3K7, Canada, and \\ ${ }^{5}$ Department of Molecular Genetics, Donnelly Centre for Cellular and Biomolecular Research, University of Toronto, Toronto, ON M5S, Canada
}

*Corresponding author: Department of Biochemistry, The University of Western Ontario, London, ON N6A 5C1, Canada. Email: mberg2@uwo.ca (M.D.B.); cbrandl@uwo.ca (C.J.B.)

${ }^{\dagger}$ These authors contributed equally to this work.

\begin{abstract}
Mistranslation, the misincorporation of an amino acid not specified by the "standard" genetic code, occurs in all organisms. tRNA variants that increase mistranslation arise spontaneously and engineered tRNAs can achieve mistranslation frequencies approaching $10 \%$ in yeast and bacteria. Interestingly, human genomes contain tRNA variants with the potential to mistranslate. Cells cope with increased mistranslation through multiple mechanisms, though high levels cause proteotoxic stress. The goal of this study was to compare the genetic interactions and the impact on transcriptome and cellular growth of two tRNA variants that mistranslate at a similar frequency but create different amino acid substitutions in Saccharomyces cerevisiae. One tRNA variant inserts alanine at proline codons whereas the other inserts serine for arginine. Both tRNAs decreased growth rate, with the effect being greater for arginine to serine than for proline to alanine. The tRNA that substituted serine for arginine resulted in a heat shock response. In contrast, heat shock response was minimal for proline to alanine substitution. Further demonstrating the significance of the amino acid substitution, transcriptome analysis identified unique up- and downregulated genes in response to each mistranslating tRNA. Number and extent of negative synthetic genetic interactions also differed depending upon type of mistranslation. Based on the unique responses observed for these mistranslating tRNAs, we predict that the potential of mistranslation to exacerbate diseases caused by proteotoxic stress depends on the tRNA variant. Furthermore, based on their unique transcriptomes and genetic interactions, different naturally occurring mistranslating tRNAs have the potential to negatively influence specific diseases.
\end{abstract}

Keywords: mistranslation; tRNA variants; synthetic genetic array; S. cerevisiae; transcriptomics

\section{Introduction}

Mistranslation, the incorporation of an amino acid not specified by the "standard" genetic code, occurs with frequencies varying from $10^{-2}$ to $10^{-5}$, depending on the codon (reviewed in Joshi et al. 2019). Mistranslation increases in specific environmental conditions (reviewed in Mohler and Ibba 2017) and due to mutations in the translation machinery, including in tRNA encoding genes (reviewed in Berg and Brandl 2021). In particular, tRNA aminoacylation plays a key role in maintaining translation fidelity, since the ribosome validates the codon-anticodon pairing and not the amino acid (Chapeville et al. 1962; Ogle et al. 2002; Loveland et al. 2017). Aminoacyl-tRNA synthetases (aaRS) recognize their cognate tRNAs using structural elements and nucleotides within the tRNA called identity elements (Rich and RajBhandary 1976; de Duve 1988; Giegé et al. 1998). For many tRNAs, recognition is determined by the anticodon. This is not the case for tRNA ${ }^{\text {Ser }}$ and tRNA $^{\text {Ala }}$ (Giegé et al. 1998). For tRNA ${ }^{\text {Ser }}$, the main identity element is the long variable arm positioned 3' of the anticodon stem (Asahara et al. 1994; Biou et al. 1994; Himeno et al. 1997). Therefore, anticodon mutations in tRNA $^{\text {Ser }}$ encoding genes lead to misincorporation of serine at nonserine codons (Geslain et al. 2010; Berg et al. 2017, 2019b; Zimmerman et al. 2018). The main identity element for tRNA ${ }^{\text {Ala }}$ is a G3: U70 base pair in the acceptor stem (Imura et al. 1969; Hou and Schimmel 1988, 1989). Addition of this element to nonalanine tRNAs results in misaminoacylation and mistranslation of alanine at nonalanine codons (McClain and Foss 1988; Francklyn and Schimmel 1989; Lant et al. 2018; Hoffman et al. 2017a).

Mutations in tRNAs that cause mistranslation arise spontaneously. Early studies identified extragenic suppressors that change the meaning of the genetic code (Crawford and Yanofsky 1959; Stadler and Yanofsky 1959; Yanofsky and Crawford 1959; Benzer and Champe 1962; Gorini and Beckwith 1966). These include tRNA variants that lead to glycine mistranslation at arginine or 
cysteine codons in Escherichia coli (Carbon et al. 1966; Gupta and Khorana 1966; Jones et al. 1966) and a tRNA ${ }^{\text {Tyr }}$ variant in yeast that suppresses stop codons (Goodman et al. 1977). Similar tRNA variants that likely mistranslate are found in human populations. In a sample of 84 individuals, we identified six variants that create G3: U70 base pairs in nonalanine tRNAs and 14 anticodon variants that alter tRNA decoding identity (Berg et al. 2019a).

Multiple copies of the tRNA genes and overlapping isoacceptors allow cells to tolerate the mistranslation caused by tRNA variants; for example, Saccharomyces cerevisiae contain approximately 275 tRNA encoding loci. In addition, cells have protein quality control mechanisms to cope with mismade proteins resulting from mistranslation. These include the ubiquitinproteasome system, autophagy, induction of the heat shock and unfolded protein responses, and the organization of aggregates into inclusion bodies (reviewed in Hoffman et al. 2017b). When mistranslation reaches a threshold, protein quality control mechanisms no longer protect the cell. Ruan et al. (2008) demonstrated that E. coli tolerate mistranslation frequencies of approximately $10 \%$. Our work suggests that yeast cells remain viable with mistranslation approaching 8\% (Berg et al. 2019b). Based on the prevalence of naturally occurring tRNA variants that potentially mistranslate, we hypothesize that mistranslation in humans could modulate disease, especially diseases characterized by loss of proteostasis (see, for example, Lee et al. 2006; Liu et al. 2014; Lu et al. 2014 and reviewed in Lant et al. 2019).

The abundance of naturally occurring tRNA variation, the relationship between tRNA variation and mistranslation and the association of mistranslation with the disease led us to investigate the genetic interactions and the impact on the transcriptome and cellular growth of different mistranslating tRNA variants in yeast. Previously, we found that repression of yeast growth caused by tRNA ${ }^{\text {Ser }}$ variants that misincorporate serine at proline codons correlated with the frequency of mistranslation (Berg et al. 2019b). However, when investigating tRNAs that mistranslate serine at a range of nonserine codons, Zimmerman et al. (2018) found that decreased growth did not simply correlate with mistranslation frequency. In this study, we compare the impact of two tRNA variants that mistranslate at similar frequencies but substitute different amino acids. Because mistranslating tRNA variants that substitute different amino acids will impact a distinct subset of cellular proteins, we hypothesize that they will have different cellular consequences in addition to causing proteotoxic stress. Using variants that mistranslate either alanine at proline codons or serine at arginine codons, we find differences in growth rate, heat shock response, transcriptomes, and genetic interactions for each of the two tRNA variants. We suggest that the nature of the amino acid substitution is important in determining the impact of human tRNA variants on diseases caused by protein misfolding, and that different tRNA variants have the potential to negatively influence distinct diseases, aside from those characterized by protein misfolding.

\section{Materials and methods Yeast strains and growth}

BY4741 (MATa his3 $\Delta 0$ leu2 $\Delta 0$ met15 $\Delta 0$ ura3 $\Delta 0$; Brachmann et al.

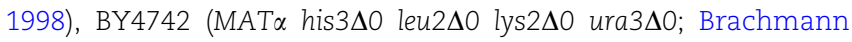
et al. 1998), and Y7092 (SGA starting strain, MAT $\alpha$ can $1 \Delta::$ STE2prSpHIS5 lyp $1 \Delta$ his $3 \Delta 1$ leu2 $\Delta 0$ ura3 $\Delta 0$ met15 $\Delta 0$ ) strains are derivatives of S288c. Y7092 was a kind gift from Dr. Brenda Andrews (University of Toronto). Strains from the temperature sensitive collection are derived from the wild-type MATa haploid yeast strain BY4741 and described in Costanzo et al. (2016). Strains containing mistranslating tRNAs were made by integrating genes encoding tRNA ${ }_{G 3: U 70}^{\text {Pro }}(C Y 8612)$ or tRNA UCU,G26A $_{\text {(CY8614) into }}^{\text {Ser }}$ Y7092 at the HO locus using the constructs described below and selecting for the natNT2 marker. The control strain (CY8611) was made by integrating only the natNT2 marker at the HO locus. Transformants were selected on YPD media containing $100 \mu \mathrm{g} / \mathrm{ml}$ nourseothricin-dihydrogen sulfate (clonNAT) and integration was verified by PCR.

Yeast strains were grown in yeast peptone (YP) media containing $2 \%$ glucose (YPD) or synthetic media supplemented with nitrogenous bases and amino acids at $30^{\circ}$. Growth curves were generated by diluting saturated cultures to $\mathrm{OD}_{600}$ equal to 0.1 and incubating at $30^{\circ}$. Growth curves were performed either in YPD, YPD containing $4 \%$ ethanol, YP containing $2 \%$ galactose, synthetic complete (SC) media containing ammonium sulfate as the nitrogen source and either $2 \%$ glucose or $2 \%$ galactose as the carbon source, SC media containing monosodium glutamate as the nitrogen source and $2 \%$ glucose as the carbon source or minimal media containing ammonium sulfate as the nitrogen source, $2 \%$ glucose, adenine, histidine, leucine, tryptophan, lysine, and methionine. $\mathrm{OD}_{600}$ was measured every 15 minutes using a BioTek Epoch 2 microplate spectrophotometer for 24 hours. Doubling time was calculated using the R package "growthcurver" (Sprouffske and Wagner 2016)

\section{DNA constructs}

Constructs to integrate mistranslating tRNAs at the HO locus were created using synthetic DNA containing $200 \mathrm{bp}$ up and downstream of the HO translational start as previously described in Zhu et al. (2020; Supplementary Figure S1; Life Technologies). The construct was cloned into pGEM ${ }^{\circledR}$-T Easy (Promega Corp.) as a NotI fragment to create pCB4386. The natNT2 marker from pFA6-natNT2 was PCR amplified using primers UK9789/UK9790 (Supplementary Table S1) and cloned into pCB4386 as an EcoRI fragment to generate the control SGA integrating vector (pCB4394). The gene encoding tRNA $\mathrm{G}_{\mathrm{G}: \mathrm{U} 70}^{\text {Pro }}$ was moved as a HindIII fragment from pCB2948 (Hoffman et al. 2017a) into pCB4394 to create pCB4396. The gene encoding tRNA $A_{U C U, G 26 A}^{\text {Ser }}$ was PCR amplified from pCB4224 (Berg et al. 2019b) using primers UG5953/ VB2609 and cloned as a HindII fragment into pCB4394 to create pCB4398.

URA3-containing centromeric plasmids expressing tRNA ${ }^{\text {Ser }}$ (pCB3076), tRNA $\mathrm{G}_{\mathrm{G} 3: \mathrm{U} 70}^{\text {ro }}$ (pCB2948), and tRNA $\mathrm{UCU}_{\mathrm{UC}, \mathrm{G} 26 \mathrm{~A}}^{\mathrm{Ser}}(\mathrm{pCB} 4301)$ are described in Berg et al. (2017), Hoffman et al. (2017a), and Berg et al. (2019b), respectively. The centromeric plasmid containing HSE-eGFP was kindly provided by Onn Brandman (Stanford University; Brandman et al. 2012).

\section{Mass spectrometry}

Liquid chromatography tandem mass spectrometry to identify mistranslation was performed on five biological replicates of the control strain (CY8611) and strains containing one of the mistranslating tRNAs (CY8612 or CY8614). Starter cultures of each strain were grown to saturation in YPD, diluted to an $\mathrm{OD}_{600}$ of 0.1 in the same media, and grown to an $\mathrm{OD}_{600}$ of $\sim 0.8$ at $30^{\circ}$. Preparation of cell lysates, protein reduction, and alkylation were performed as described in Berg et al. (2019b). Robotic purification and digestion of proteins into peptides were performed on the KingFisher ${ }^{\mathrm{TM}}$ Flex using LysC and the R2-P1 method as described in Leutert et al. (2019). 
Peptides were analyzed on a hybrid quadrupole orbitrap mass spectrometer (Orbitrap Exploris 480; Thermo Fisher Scientific) equipped with an Easy1200 nanoLC system (Thermo Fisher Scientific). Peptide samples were resuspended in $4 \%$ acetonitrile, $3 \%$ formic acid and loaded onto a $100 \mu \mathrm{m} \mathrm{ID} \times 3 \mathrm{~cm}$ precolumn packed with Reprosil C18 $3 \mu \mathrm{m}$ beads (Dr. Maisch GmbH), and separated by reverse-phase chromatography on a $100 \mu \mathrm{m}$ ID $\times$ $30 \mathrm{~cm}$ analytical column packed with Reprosil C18 $1.9 \mu \mathrm{m}$ beads (Dr. Maisch $\mathrm{GmbH}$ ) housed into a column heater set at $50^{\circ}$.

Peptides were separated using a gradient of 5-30\% acetonitrile in $0.125 \%$ formic acid at $400 \mathrm{~nL} / \mathrm{min}$ over 95 minutes, with a total 120 minutes acquisition time. The mass spectrometer was operated in data-dependent acquisition mode with a defined cycle time of 3 seconds. For each cycle, one full mass spectrometry scan was acquired from 350 to $1200 \mathrm{~m} / \mathrm{z}$ at 120,000 resolution with a fill target of 3E6 ions and automated calculation of injection time. The most abundant ions from the full MS scan were selected for fragmentation using $2 \mathrm{~m} / \mathrm{z}$ precursor isolation window and beam-type collisional-activation dissociation (HCD) with $30 \%$ normalized collision energy. MS/MS spectra were acquired at 15,000 resolution by setting the AGC target to standard and injection time to automated mode. Fragmented precursors were dynamically excluded from selection for 60 seconds.

MS/MS spectra were searched against the S. cerevisiae protein sequence database (downloaded from the Saccharomyces Genome Database resource in 2014) using Comet (release 2015.01; Eng et al. 2013). The precursor mass tolerance was set to $50 \mathrm{ppm}$. Constant modification of cysteine carbamidomethylation $(57.0215 \mathrm{Da})$ and variable modification of methionine oxidation (15.9949 Da) were used for all searches. Variable modification of proline to alanine $(-26.0157 \mathrm{Da})$ or arginine to serine $(-69.0691 \mathrm{Da})$ were used for the respective mistranslating tRNAs. A maximum of two of each variable modification were allowed per peptide. Search results were filtered to a $1 \%$ false discovery rate at the peptide spectrum match level using Percolator (Käll et al. 2007). The mistranslation frequency was calculated using the unique mistranslated peptides for which the nonmistranslated sibling peptide was also observed. The frequency is defined as the counts of mistranslated peptides, where alanine was inserted for proline or serine inserted for arginine, divided by the counts of all peptides containing proline or arginine, respectively, and expressed as a percentage.

\section{Synthetic genetic array analysis and validation}

The synthetic genetic array (SGA) assay was performed as described by Tong et al. (2001) with minor modifications. Strains

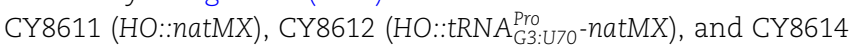
(HO:: tRNA $A_{U C U, G 26 A}^{\text {Ser }}$ natMX) were mated with a yeast temperature sensitive collection (Ben-Aroya et al. 2008; Li et al. 2011; Kofoed et al. 2015; Costanzo et al. 2016) in quadruplicate 1536 colony array format using a BioMatrix (S\&P Robotics Inc.) automated pinning system. In this format, each allele of the temperature sensitive collection is present in biological quadruplicate on the plate. Double mutants were selected on YPD plates containing $200 \mathrm{mg} / \mathrm{L}$ G418 and $100 \mathrm{mg} / \mathrm{L}$ clonNAT. Diploids were sporulated on enriched sporulation media and MATa haploid doublemutants selected using standard SGA media. To identify genetic interactions, double mutants were pinned onto double mutant selection SGA medium and grown at $30^{\circ}$ for 5 days. Images were taken every 24 hours to determine colony size computationally. SGATools (Wagih et al. 2013) was used to determine genetic interaction scores using a multiplicative model. Double mutant strains with average interaction score less than -0.2 and
Benjamini-Hochberg corrected P-value less than 0.05 were considered as potential negative genetic interactions.

Double mutants that were identified as negative genetic interactions from the screen were validated by re-creating the double mutant strain, starting from the single mutant haploid strains, using the SGA approach. Double mutant strains were grown in liquid media to saturation, cell densities were normalized, and cultures were spotted on SGA media. The temperature sensitive mutant crossed with the control strain CY8611 and the mistranslating tRNA strain crossed with a control his $3 \Delta$ strain were also spotted to determine fitness of the single mutants. Intensity of each spot was measured with ImageJ (Schneider et al. 2012). Expected double mutant growth was calculated based on the growth of the single mutants and compared to the experimental growth of the double mutant. Double mutants that grew more slowly than expected were considered validated negative genetic interactions. Raw and validated data can be found in Supplementary File S2.

\section{RNA preparation, sequencing, and analysis}

Wild-type yeast strain BY4742 containing either an empty URA3 plasmid (control), pCB2948 (tRNA $A_{\mathrm{G} 3: U 70}^{\text {Pro }}$ ), or pCB4301 (tRNA UCU,G26A $_{\text {Ser }}$ ) were grown to stationary phase in media lacking uracil. Strains were diluted in the same media to an $\mathrm{OD}_{600}$ of 5.0 $\times 10^{-3}$ and grown to a final $\mathrm{OD}_{600}$ of 2.0. Cells were harvested and RNA extracted with MasterPure ${ }^{\mathrm{TM}}$ Yeast RNA Purification Kit (Epicentre). Samples were vacuum dried and sent to Genewiz (South Plainfield, NJ, USA) for total RNA sequencing.

Stranded Illumina TruSeq cDNA libraries with poly dT enrichment were prepared from high quality total RNA (RIN > 8). Libraries were sequenced on an Illumina HiSeq yielding between 30.2 and 39.5 million 150 bp paired-end reads per sample.

FASTQ files were analyzed using a customized bioinformatics workflow. Adapter sequences and low-quality bases were trimmed using the default settings of Trimmomatic (Bolger et al. 2014). Sequence quality was analyzed using FastQC (http://www. bioinformatics.babraham.ac.uk/projects/fastqc/)(Last accessed October 1, 2020). Reads were aligned to the S. cerevisiae S288C reference genome (Engel et al. 2014; release R64-2-1_20150113) using STAR (Dobin et al. 2013). Only uniquely mapping reads were counted. Read counts for each gene were summarized using featureCounts (Liao et al. 2014). Differential expression analysis was performed using the DESeq2 R package (Love et al. 2014) with a Benjamini-Hochberg adjusted P-value cut off of $\leq 0.05$. Analysis script can be found in Supplementary File S3. The data have been deposited in NCBI's Gene Expression Omnibus (Edgar et al. 2002) and are accessible through GEO Series accession number GSE174145.

\section{GO term analysis and genetic interaction network creation}

Gene ontology (GO) term analysis was performed using the GO term finder tool (http://go.princeton.edu/) using a P-value cut-off of 0.01 after applying Bonferroni correction. Terms were filtered with REVIGO (Supek et al. 2011). Networks were constructed using Cytoscape 3.7 (Shannon et al. 2003).

\section{Heat shock assay}

Yeast strains containing the HSE-GFP reporter (Brandman et al. 2012) and a mistranslating tRNA variant were grown to stationary phase in medium lacking uracil and containing $0.6 \%$ casamino acids, diluted 1:100 in the same medium and grown for 18 hours at $30^{\circ}$. Cell densities were normalized to $\mathrm{OD}_{600}$ before 
measuring fluorescence with a BioTek Synergy H1 microplate reader at an emission wavelength of $528 \mathrm{~nm}$. The mean relative fluorescence units were calculated from three technical replicates for each biological replicate.

\section{Results}

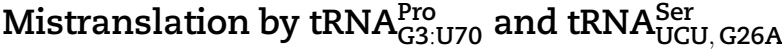

Our goal was to compare the impact of two tRNA variants that mistranslate at a similar frequency, calculated from the ratio of mistranslated peptides to peptides containing the wild-type residue, but cause different amino acid substitutions. Previously, we have identified yeast tRNAs that mistranslate alanine or serine at different codons (Berg et al. 2017, 2019b; Hoffman et al. 2017a). Our quantification of their mistranslation frequencies using mass spectrometry led us to further analyze two tRNAs (Figure 1A). The first tRNA variant, tRNA $\mathrm{G}^{\text {Pro }}$ U70, contains a G3: U70 base pair in the acceptor stem of a proline tRNA and mistranslates alanine at proline codons (Hoffman et al. 2017a). The second tRNA variant, tRNA $\mathrm{UCU}_{\mathrm{UCU}, \mathrm{G} 26 \mathrm{~A}}$, is a serine tRNA with an arginine anticodon and a G26A secondary mutation to dampen tRNA function and mistranslates serine at arginine codons (Berg et al. 2017, 2019b). As one of the planned comparisons was an SGA analysis, we constructed strains where the gene encoding the tRNA variants was integrated into the genome at the HO locus and selected with a clonNAT resistance marker. A third strain was created as a control with the clonNAT resistance marker alone integrated at the HO locus.

Using mass spectrometry, we determined the frequency of mistranslation for each strain (Figure 1B; Supplementary Table S2). The frequency of proline to alanine mistranslation in the strain expressing tRNA $\mathrm{G}_{\mathrm{G} 3: \mathrm{U} 70}^{\mathrm{Pr}}$ Was 3.1\%. Arginine to serine substitution in the strain expressing tRNA $\mathrm{UCU}_{\mathrm{UCU}, \mathrm{G} 26 \mathrm{~A}}$ was a similar $3.0 \%$. The corresponding substitutions occurred at a frequency of approximately $0.2 \%$ in the control strain. Based on the mistranslated peptides identified from the proteomic mass spectrometry analysis of the proteome, $\mathrm{tRNA}_{\mathrm{G} 3: \mathrm{U} 70}^{\mathrm{Pro}}$ predominantly mistranslated alanine at
CCA proline codons, whereas tRNA $A_{U C U, G 26 A}^{\text {er }}$ decoded primarily AGA arginine codons (Supplementary Figure S2).

\section{Characterizing the impact of mistranslation on cellular phenotypes}

We determined the effect of the two tRNAs on cell growth in liquid culture under various nutrient conditions: YP or SC media with glucose or galactose as the carbon source, YP with glucose containing 4\% ethanol, SC with monosodium glutamate and glucose as the nitrogen and carbon sources, respectively, and minimal media containing ammonium sulfate as the nitrogen source, $2 \%$ glucose, adenine, histidine, leucine, lysine, and methionine. Both strains expressing mistranslating tRNAs grew slower than the control strain in all conditions (Figure 2A). These differences were statistically significant $(P \leq 0.05$; Welch's $t$-test) with the exception of the strain expressing tRNA ${ }_{G 3: U 70}^{\text {Pro }}$ grown in medium containing $4 \%$ ethanol. In rich media (YPD), the alanine mistranslating tRNA (tRNA ${ }_{\mathrm{G} 3: \mathrm{U} 70}^{\text {Pro }}$ ) increased doubling time to 80 minutes as compared to 74 minutes for the control strain. The serine mistranslating tRNA (tRNA $\mathrm{SCU}$ Ser $\left.{ }_{\text {G } 6 \mathrm{~A}}\right)$ had a somewhat greater impact on growth, increasing doubling time to 85 minutes. This trend of the arginine to serine substitution having a greater effect was seen under all conditions, except in rich media containing galactose as the carbon source (YP + Galactose) where the two mistranslating tRNAs similarly increased doubling time relative to the control. Also indicative of the effect of environment on the phenotypic consequences of specific mistranslating tRNAs, the negative impact of $\mathrm{tRNA}_{\mathrm{UCU}, \mathrm{G} 26 \mathrm{~A}}^{\mathrm{Ser}}$ was greater when cells were grown in SC media with either glucose or galactose as the carbon source.

Mistranslation results in proteotoxic stress and a heat shock response (Berg et al. 2017). To determine the level of heat shock response found in the strains containing the tRNA variants, we measured fluorescence arising from GFP under the control of a synthetic heat shock promoter (HSE; Brandman et al. 2012; Figure 2B). Expression of HSE-GFP was 2.8-fold greater in cells containing tRNA $\mathrm{UCU}_{\mathrm{UCU}, \mathrm{G} 26 \mathrm{~A}}^{\mathrm{Ser}}(\mathrm{Arg} \rightarrow \mathrm{Ser})$ than in the control strain. In contrast, expression of HSE-GFP in RRNA $_{\mathrm{G} 3: \mathrm{U} 70}^{\text {Pro }}$ (Pro $\rightarrow$ Ala) was not statistically different from the control.
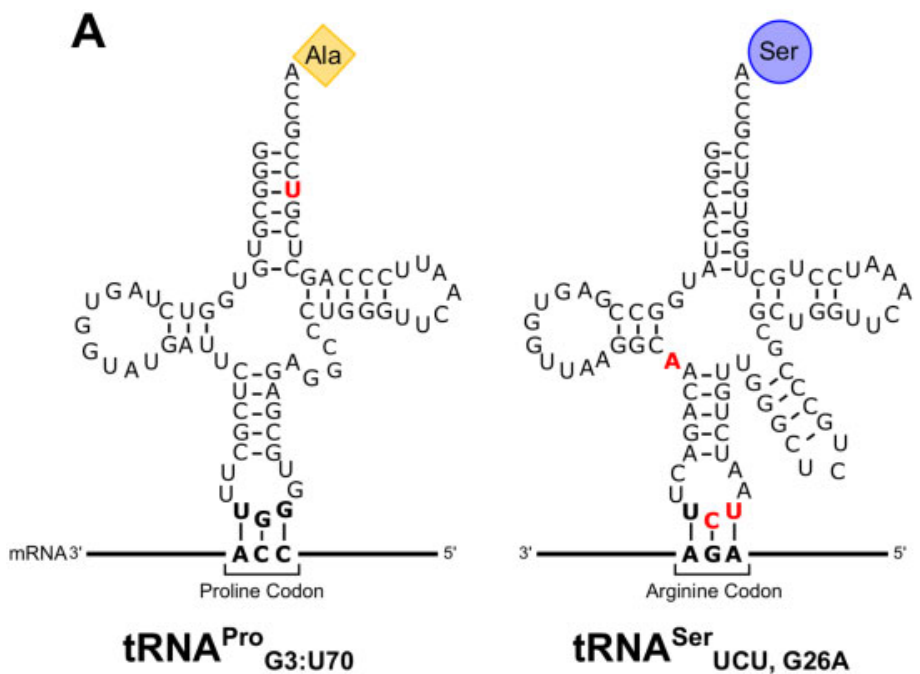

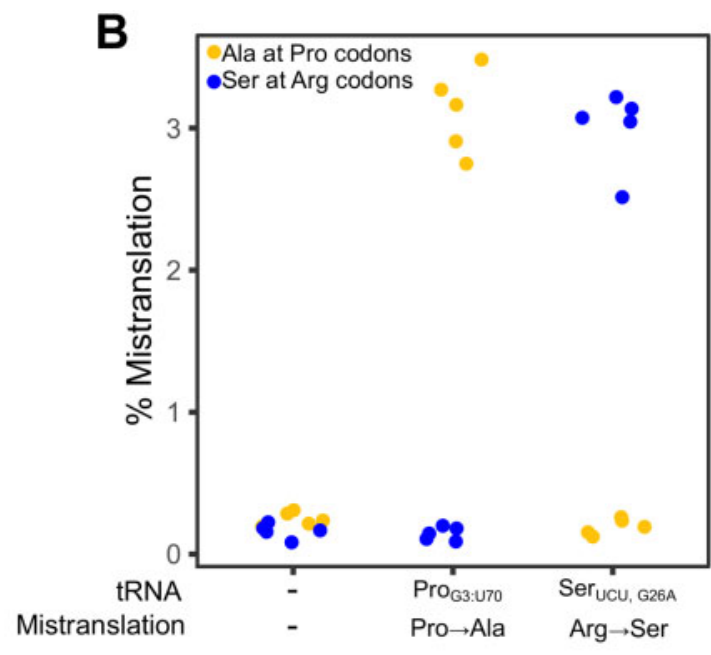

Figure 1 tRNA variants that mistranslate. (A) Secondary structure of $\mathrm{tRNA}_{\mathrm{G} 3: \mathrm{U} 70}^{\mathrm{Pro}}$, which mistranslates alanine at proline codons, and tRNA $\mathrm{UCU}_{\mathrm{G}, \mathrm{G} 26 \mathrm{~A}}$, which mistranslates serine at arginine codons. Nucleotides colored in red denote differences compared to the wild-type tRNA ${ }^{\text {Pro }}$ or tRNA ${ }^{\text {Ser }}$, respectively. (B) Mass spectrometry analysis of the cellular proteome was performed on a control strain with no additional tRNAs (CY8611) or strains expressing mistranslating tRNA variants tRNA $\mathrm{GrO}$ :U70 (CY8612) or tRNA $\mathrm{UCr}_{\mathrm{UCU}, \mathrm{G} 26 \mathrm{~A}}^{\mathrm{S}}$ (CY8614). Each point represents one biological replicate. Each strain expressing a mistranslating tRNA had statistically higher frequency of mistranslation compared to the control strain ( $P \leq 0.05$; Welch's t-test). 

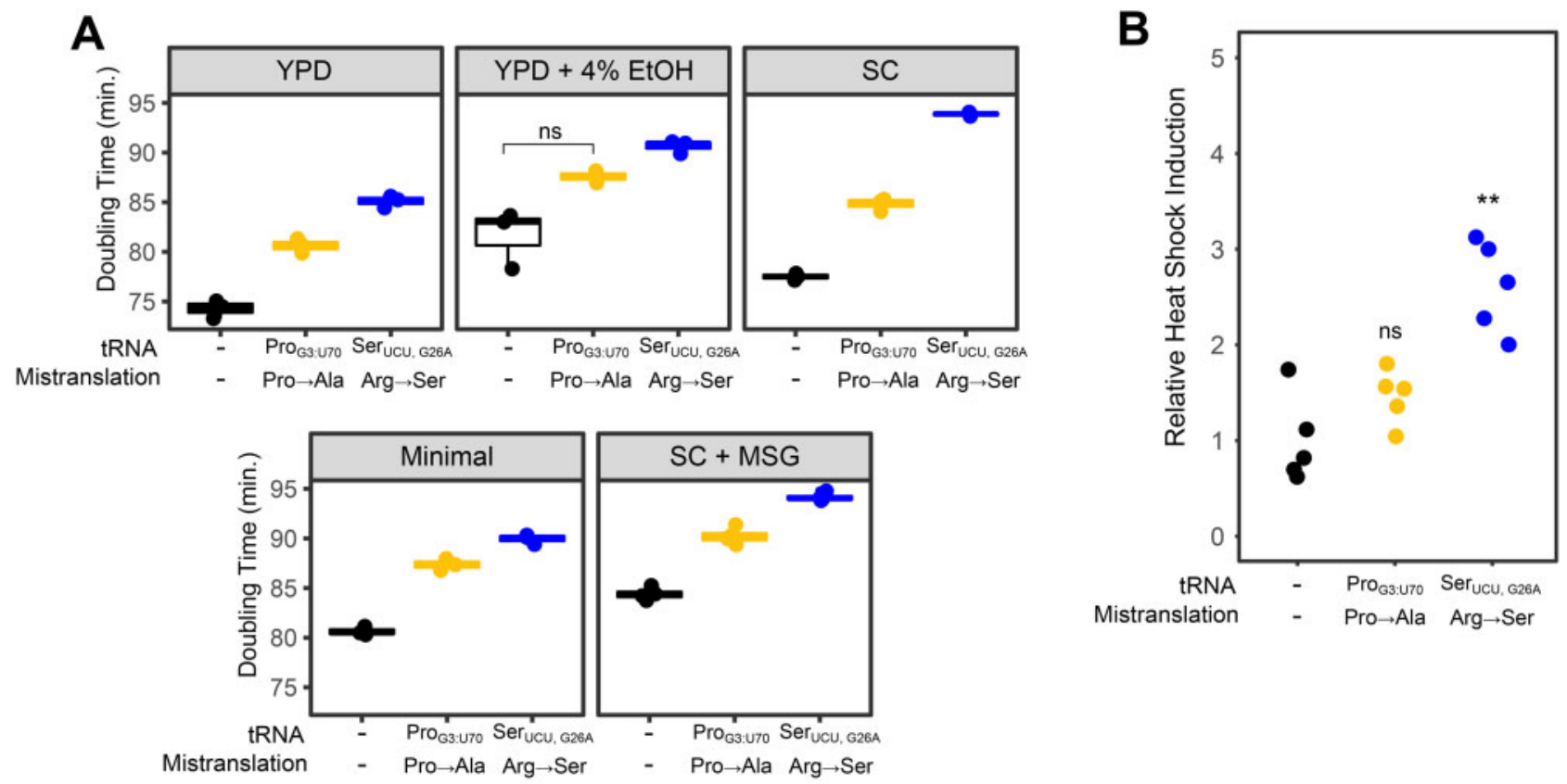

Mistranslation - Pro $\rightarrow$ Ala $\quad \mathrm{Arg} \rightarrow \mathrm{Ser}$

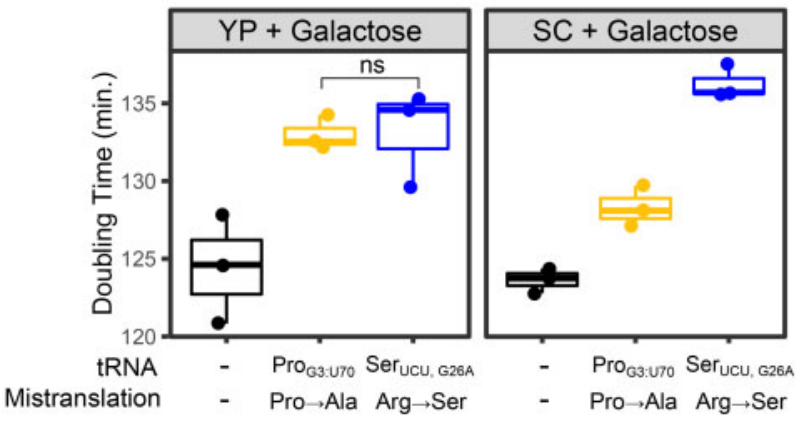

Figure 2 Phenotypic characterization of mistranslating strains. (A) Growth rates for either a control strain with no additional tRNA (CY8611) or strains expressing mistranslating tRNA variants tRNA ${ }_{G 3: U 70}^{\text {Pro }}$ (CY8612) or tRNA $A_{U C U . G 26 A}^{\text {Ser }}$ (CY8614) were determined from growth curves of the strains diluted to an $\mathrm{OD}_{600} \sim 0.1$ in various media and grown for 24 hours. (YPD-YP with $2 \%$ glucose, YP + Galactose-YP with 2\% galactose, SC-synthetic complete with ammonium sulfate as the nitrogen source and $2 \%$ glucose or $2 \%$ galactose as the carbon source, minimal-medium containing ammonium sulfate as the nitrogen source, $2 \%$ glucose and adenine, histidine, leucine, lysine, and methionine, SC + MSG — synthetic complete media with monosodium glutamate and glucose as nitrogen and carbon sources). Doubling time was calculated with the R package "growthcurver" (Sprouffske and Wagner 2016). Each point represents one biological replicate. All comparisons within a growth condition are statistically different (Bonferroni corrected $P \leq 0.05$; Welch's t-test), except where indicated (ns = not significant). (B) Strains described in A were transformed with a GFP reporter transcribed from a promoter containing heat shock response elements, grown to saturation in media lacking uracil, diluted 1:100 in the same media and grown for 18 hours at $30^{\circ}$. Cell densities were normalized and fluorescence measured. Each point represents one biological replicate. Statistical comparisons were made between strains expressing a variant tRNA and the control strain (ns = not significant, ${ }^{* *} \mathrm{P} \leq 0.005$; Welch's t-test).

\section{Mistranslating tRNAs have different genetic interactions}

We used a SGA analysis to identify the genetic interactions of the two tRNAs and provide another comparison of the impact of the substitutions. Preliminary screens of the strain expressing tRNA $A_{G 3: U 70}^{\text {Pro }}$ identified a greater percentage of interactions with the temperature sensitive collection than the deletion collection, therefore the former was chosen for the comparison of

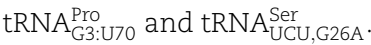

SGA analyses were performed in parallel for the mistranslating and control strains. Of the 1016 alleles in the temperature sensitive collection, 18 had a negative genetic interaction with $\mathrm{tRNA}_{\mathrm{G} 3: \mathrm{U} 70}^{\text {Pro }}($ Pro $\rightarrow$ Ala), whereas 78 alleles had a negative interaction with $\mathrm{tRNA}_{\mathrm{UCU}, \mathrm{G} 26 \mathrm{~A}}^{\mathrm{Ser}}(\mathrm{Arg} \rightarrow \mathrm{Ser}$ ). No positive genetic interactions were identified. Genetic interactions were validated by remaking the double mutant strains, spotting normalized densities of the double mutants and their control strain on selective medium, and measuring growth after 2 days. After validation, 10 and 47 alleles showed negative genetic interactions with $\mathrm{tRNA}_{\mathrm{G} 3: \mathrm{U70}}^{\mathrm{Pro}}$ and $\mathrm{tRNA}_{\mathrm{UCU}, \mathrm{G} 26 \mathrm{~A}}^{\mathrm{Ser}}$, respectively (Figure 3). The increased number of genes identified with $\mathrm{tRNA}_{\mathrm{UCU}, \mathrm{S} 26 \mathrm{~A}}^{\mathrm{Ser}}$ parallels its greater effect on growth and greater heat shock response. Raw data and validated genes are found in Supplementary File S2.

Analysis of the biological function of the genes displaying negative genetic interactions with the mistranslating tRNA variants revealed both distinct and common processes. Not surprisingly, many of the processes were also identified in chemical-genetic screens with amino acid analogs that are incorporated into protein (Berg et al. 2020). As mentioned above, the most notable difference in the genetic interaction patterns between the two tRNAs is the increased number with tRNA $\mathrm{UCU}, \mathrm{Se} 26 \mathrm{~A}$. The difference in number of interactions makes evaluating whether there is enrichment for one of the tRNAs difficult. In general, both tRNAs have genetic interactions with genes involved in transcription and protein quality control. The former suggests a potential importance for a transcriptional response to mistranslation, while 


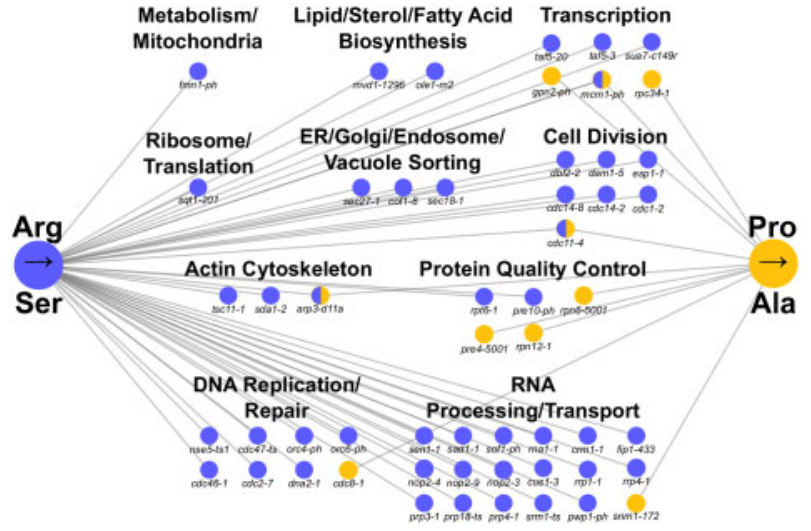

Figure 3 Negative genetic interaction networks of the mistranslating tRNAs. Genetic interaction network of temperature sensitive alleles that have negative genetic interactions with tRNA $\mathrm{G}_{\mathrm{G} 3: \mathrm{U} 70}^{\text {Pro }}$ (yellow) and tRNA $\mathrm{UCU}_{\mathrm{UCU}}^{\mathrm{Ser}}$ (blue). Nodes represent alleles and edges represent negative genetic interactions.

the later highlights the role of proteasomal components in coping with mismade proteins (Shcherbakov et al. 2019). While a negative genetic interaction with a mistranslating tRNA variant may indicate the gene/process is required for cells to cope with the resulting mismade protein, it may also indicate that the process is sensitive to specific mistranslation. For example, 15 of the 16 genes involved in RNA processing and transport were specifically found with $\mathrm{tRNA}_{\mathrm{UCU}, \mathrm{G} 26 \mathrm{~A}}^{\mathrm{Ser}}$ suggesting the possibility that the individual proteins or the process may be particularly sensitive to substitution at arginine codons. The RNA processing/transport genes provide an example that highlights that although processes were shared, specific genetic interactions differed. The mistranslating tRNAs only shared negative genetic interactions with three alleles. This led us to evaluate whether amino acid composition of the encoded protein contributed to a gene being synthetic with a mistranslating tRNA, using the RNA processing/transport genes as a test case. The average arginine content of the proteins whose genes are synthetic with tRNA $\mathrm{UCU}_{\mathrm{UCU}, \mathrm{G} 26 \mathrm{~A}}^{\mathrm{Ser}}$ varies from $7.8 \%$ (Sof1) to $2.5 \%$ (Rna1) as compared to the cellular average of $4.4 \%$ (Supplementary Table S3). In several cases, the arginine content of the tRNA $\mathrm{UCr}_{\mathrm{UC}, \mathrm{G} 26 \mathrm{~A}}^{\mathrm{Sin}}$ ieractors is less than the proline content (Sad1, Rna1, Fip1, Pwp1, and Srm1). This indicates that amino acid composition of the encoded proteins alone does not explain the specific negative genetic interactions with these genes.

\section{Impact of tRNA ${ }_{\mathrm{G} 3: \mathrm{U} 70}^{\mathrm{Pro}}$ or tRNA $\mathrm{UCU}_{\mathrm{UC}, \mathrm{G} 26 \mathrm{~A}}^{\mathrm{Ser}}$ on the transcriptome}

We next compared the transcriptome profiles of strains expressing tRNA $A_{G 3: U 70}^{\text {Pro }}$ or tRNA $\mathrm{UCCU}_{\mathrm{U}, \mathrm{G} 26 \mathrm{~A}}$. To ensure that the strains had identical genetic backgrounds without possible suppressor mutations, we transformed plasmids expressing the mistranslating tRNAs or empty plasmid into yeast strain BY4742. The transcriptome of each strain was profiled using an RNA sequencing approach. Principal component analysis (PCA) of the three transcriptome profiles demonstrated that $45 \%$ of the variance could be explained in two components (Figure 4A). The wild-type control strain clusters separately from the strains expressing mistranslating tRNAs in the first component and the two strains expressing different mistranslating tRNAs separate in the second component. This suggests that mistranslation of alanine at proline codons initiates a distinct response from mistranslation of serine at arginine codons.
We then analyzed the differentially expressed genes in each mistranslating strain as compared to the control strain. A total of 2897 genes (44.7\%) were differentially expressed in at least one strain (Benjamini-Hochberg adjusted P-value cut off $\leq 0.05$ ). A heatmap of the fold-change in transcript levels for these genes is shown in Figure 4B. A Venn diagram of the overlap of up- and down-regulated genes caused by the mistranslating tRNAs is shown in Figure 4C. Of note, distinct groups of genes with unique responses based on the type of mistranslation were identified. For example, genes involved in cell cycle, DNA replication, transcription, and response to stress were down-regulated predominantly in the strain expressing tRNA $\mathrm{G}_{\mathrm{G} 3: \mathrm{U} 70}^{\text {Pro }}$ (Pro $\rightarrow$ Ala) whereas genes involved in response to chemical and protein localization to the endoplasmic reticulum were upregulated. Genes involved in carboxylic acid biosynthetic processes and purine metabolism were upregulated predominantly in the strain expressing tRNA UCU,G26A $_{\text {Ser }} \rightarrow$ Ser). There were also common sets of genes differentially expressed in both mistranslating strains. For example, genes involved in mitochondrial translation, cell division, ribosome biogenesis and RNA metabolism were downregulated, and genes involved in carbohydrate metabolism and proteolysis were upregulated in both strains. Overall, these results demonstrate that while a common set of genes respond to mistranslation, each of the two mistranslating tRNAs induce a unique transcriptional response.

A more stringent analysis of the transcriptional changes, considering only genes with greater than twofold change in expression, revealed 55 and 78 genes up-regulated and 34 and 71 genes down-regulated in strains expressing $\mathrm{tRNA}_{\mathrm{G} 3: \mathrm{U} 70}^{\text {Pro }}$ and tRNA $\mathrm{UCr}_{\mathrm{UCU}, \mathrm{G} 26 \mathrm{~A}}$, respectively (Figure 4, D and E). Among the upregulated genes, 36 are common to both mistranslating tRNA variants. These included genes involved in carbohydrate metabolism, mainly glycogen and trehalose biosynthesis (e.g., GLC3, IGD1, GSY1, GSY2, MRK1, PGM2, PIG2, GIP2, TPS2, TPS2, and TSL1). The upregulation of genes involved in the synthesis of these storage carbohydrates is consistent with previous studies demonstrating that glycogen and trehalose accumulate under the stress of nutrient starvation or heat shock (Lillie and Pringle 1980; Hottiger et al. 1987; Parrou et al. 1997) and that trehalose stabilizes proteins and suppresses aggregation (Singer and Lindquist 1998). Genes involved in protein folding were also upregulated in both strains. These include the small heat shock protein, HSP42, which suppresses misfolded protein aggregation, components of the chaperonin complex (TCP1), and genes that direct ubiquitination of misfolded proteins (ROQ1). Five genes (FET3, AAD15, GFD2, YOR338W, and ALD6) were downregulated in both strains expressing mistranslating tRNA variants.

\section{Discussion}

Mistranslation can generate many distinct substitutions that differ with regard to the amino acid (or stop codon) replaced and the amino acid that is inserted. Previously, we found that the frequency of mistranslation resulting from tRNA variant impacts the biological consequences with increasing frequencies of mistranslation having more severe effects (Berg et al. 2019b). Here, we show that two tRNA variants that mistranslate different codons with a frequency of $\sim 3 \%$ elicit different effects on growth, heat shock response and the transcriptome and have distinct sets of genetic interactions. 

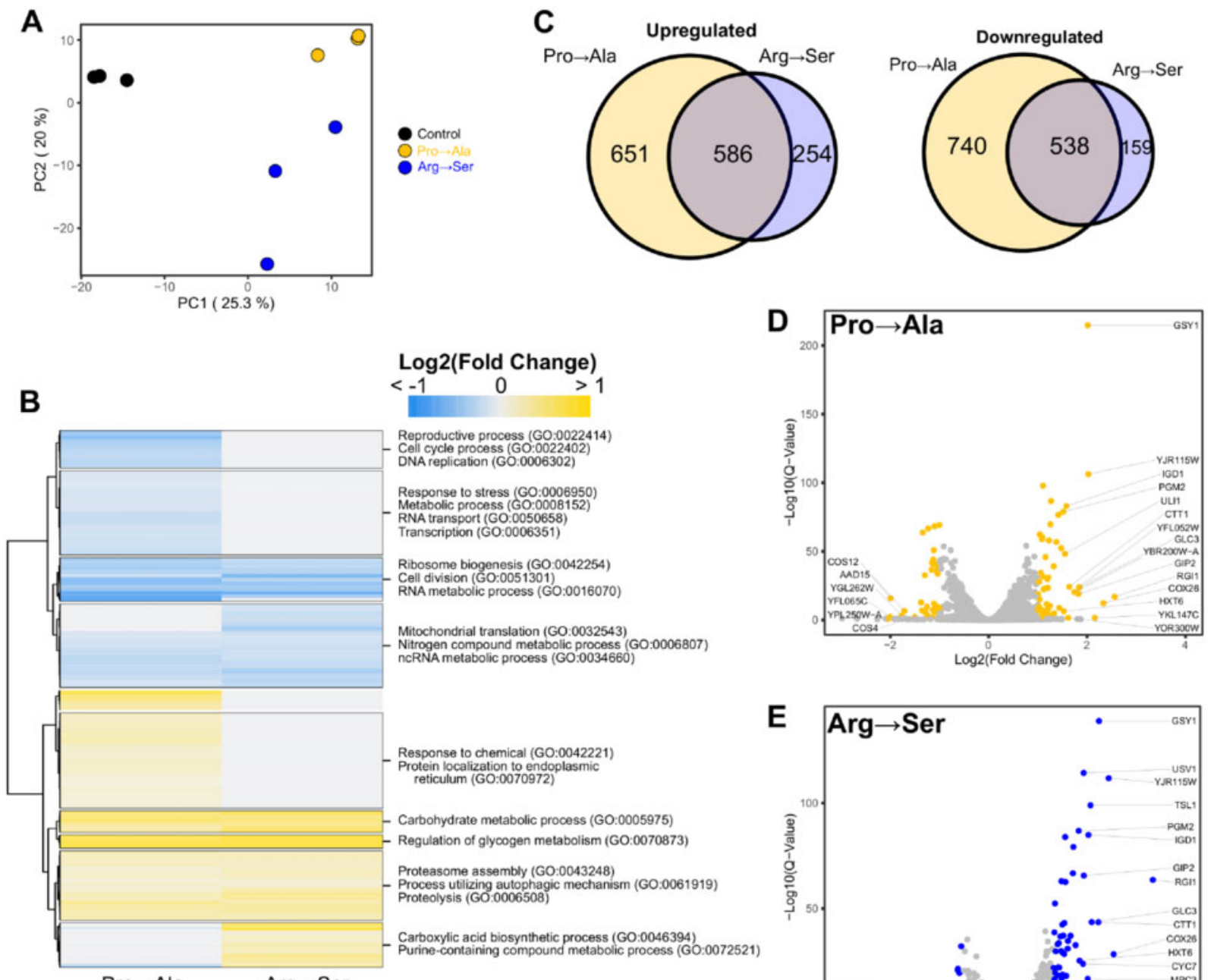

ncRNA metabolic process (GO:0034660)

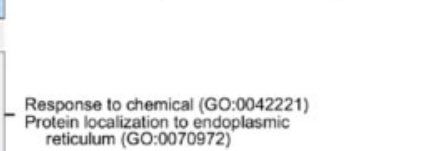

rotein localization to endoplasmic

Carbohydrate metabolic process (GO:0005975)

Regulation of glycogen metabolism (GO:0070873)

Proteasome assembly (GO:0043248)

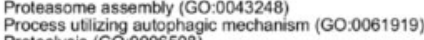

Process utilizing autophagic
Proteolysis (G0:0006508)

Carboxylic acid biosynthetic process (GO:0046394)
Purine-containing compound metabolic process (GO:0072521)

Pro $\rightarrow$ Ala

$\mathrm{Arg} \rightarrow \operatorname{Ser}$

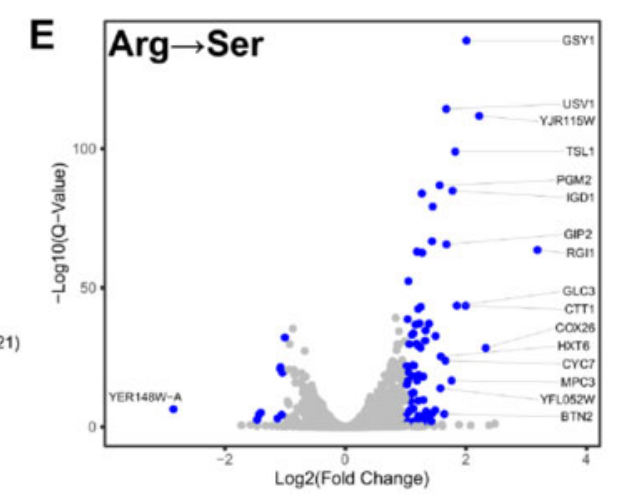

Figure 4 Transcriptome analysis of strains expressing mistranslating tRNA variants. (A) PCA of centered log ratio normalized reads from BY4742

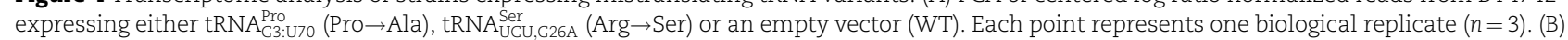
Heatmap of hierarchical clustered differentially expressed genes $(P \leq 0.05)$ in response to mistranslation. Fold-change for each gene is the average from three biological replicates. Upregulated genes are colored yellow while downregulated genes are colored blue. Significantly enriched GO terms within each cluster are annotated. (C) Venn diagram of upregulated and downregulated genes $(P \leq 0.05)$ in yeast strain BY4742 transformed with a centromeric plasmid expressing tRNA $\mathrm{Gro}$ :U70 (Pro $\rightarrow$ Ala) or tRNA Ser $\mathrm{UCH}_{\mathrm{G} 26 \mathrm{~A}}(\mathrm{Arg} \rightarrow \mathrm{Ser}$ ) as compared to BY4742 transformed with empty vector. (D) Volcano plot highlighting in yellow differentially expressed genes with greater than twofold changes in $\mathrm{tRNA}_{\mathrm{G} 3: \mathrm{U} 70}^{\text {Pro }}$ relative to the control strain. Points with gene name labels have the largest fold-change relative to control. (E) Volcano plot highlighting in blue differentially expressed genes with greater than

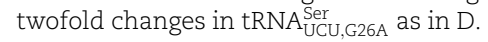

\section{Significance of the differing impacts of tRNA variants}

tRNA variants with the potential to mistranslate are found in the human population (Berg et al. 2019a). Approximately 20\% of all individuals have either an anticodon mutation in $\mathrm{tRNA}^{\text {Ser }}$ or tRNA $^{\text {Ala }}$ or an acceptor stem mutation that generates a G3: U70 base pair, the major identity element for charging with alanine (Hou and Schimmel 1988; Francklyn and Schimmel 1989). The ability of mutations in other parts of the tRNA, such as the D-arm (Hirsh 1971), to alter decoding specificity suggests that more mistranslating variants may exist amongst the more than $600 \mathrm{hu}$ man tRNA encoding genes. We hypothesize that mistranslating tRNAs are genetic modifiers of disease by increasing the level of proteotoxic stress. This increased stress could lead to the earlier onset and/or increased severity of diseases that have proteotoxic stress as their hallmark, including many neurodegenerative diseases, cardiomyopathies, and hearing loss (Willis and Patterson 2013; Labbadia and Morimoto 2015; McLendon and Robbins 2015; Jongkamonwiwat et al. 2020). In addition, mistranslating tRNA variants may also play a role in cancer by enhancing tumor growth, as demonstrated by Santos et al. (2018). In this framework, our results suggest that even for tRNA variants with a similar mistranslation frequency, their impact on the disease would differ based on the specific substitution. For example, because of the increased heat shock observed for tRNA $\mathrm{UCU}_{\text {UC,G26A }}^{\text {Ser }}$ (Arg $\rightarrow$ Ser), we expect $t R N A_{U C U, G 26 A}^{S e r}$ to have a greater impact than tRNA $A_{G 3: U 70}^{\text {Pro }}$ (Pro $\rightarrow$ Ala). Moreover, given that diseases often result from one genetic change or a polygenic mix of genetic changes, the finding 
that mistranslating tRNAs show unique patterns of negative synergy with specific genes is a strong indication that tRNA variants could act as genetic modifiers of distinct diseases. The synergy between a tRNA variant and a cellular mutation can act in either direction. Most commonly the mistranslating tRNA will alter the severity of a mutation in another gene. However, if a gene regulates tRNA expression or function, its mutation could change the impact of the mistranslating tRNA variant.

\section{Factors affecting the impact of mistranslating tRNAs}

Before addressing what alters the impact of a mistranslating tRNA, it is important to consider how proteome-wide mistranslation could affect a cell. First, as indicated above, mistranslation results in protein misfolding and aggregation that in turn give rise to proteotoxic stress. On its own, this will impact cell function. Second, decreased expression of individual cellular proteins often has a phenotypic consequence (Breslow et al. 2008). Mistranslation acts at the proteome level to decrease the amount of each protein that is functional, effectively creating hypomorphs. Global mistranslation may exaggerate the effect because of the potential that multiple genes in a pathway as well as those in redundant pathways can be compromised.

Given equal frequencies of substitution, the relative impact of two mistranslating tRNAs is determined by a number of factors. The first to consider is the absolute number of amino acid changes. Variants that mistranslate amino acids found more abundantly in the proteome have more targets for misincorporations and thus a greater probability of inducing protein unfolding and/or affecting a hypomorphic gene. In addition, amino acid composition varies from protein to protein. Therefore, each mistranslating tRNA has the potential to impact a different subset of proteins, whose amino acid composition and level of expression differ. The combination of these factors makes predicting the absolute number of amino acid substitutions difficult, especially since protein expression depends on environmental conditions (Navarrete-Perea et al. 2021). Furthermore, the structural context of the target amino acid is dependent on the individual protein, thus mistranslation affects stability and function in a proteinspecific manner.

The properties of the amino acids being substituted are the second major factor affecting the impact of proteome-wide mistranslation. In theory, more chemically diverse substitutions should have more profound effects on protein structure and function. In fact, the genetic code is predicted to have evolved by minimizing the impact of mutations on the resulting change in these properties (Koonin and Novozhilov 2017). Woese et al. (1966) proposed the polar requirement (PR) scale for the relatedness of amino acids. The PR scale is based on the solubility of each amino acid in pyridine: proline, alanine, serine, and arginine score 6.6, 7.0, 7.5, and 9.1, respectively. For mistranslating tRNAs, the substitution would be expected to be less severe if the amino acids are close on the PR scale. Fitting with this we see less impact for Pro $\rightarrow$ Ala than Arg $\rightarrow$ Ser. The distinct biochemical properties of each amino acid may also confer special functions to a protein that are lost or gained upon substitution; for example, the phosphorylation of serine and the prevalence of arginine in nuclear localization signals. We considered substitution matrices as another way to evaluate the impact of mistranslation. Substitution matrices describe the likelihood that one amino acid would be substituted for another over evolutionary time. Point accepted mutation (PAM) matrices extrapolate from alignments of closely related sequences (Dayhoff and Eck 1968), whereas blocks substitution matrix (BLOSUM) reflects changes found in more distantly related proteins (Henikoff and Henikoff 2000). We found that the substitution scores in these matrices do not match the magnitude of biological effect seen for the substitutions tested here. This may be because the matrices are compiled from evolutionarily related protein sequences where certain substitutions are less likely to occur because of genetic code constraints, rather than functional properties of the protein.

We note that we have restricted our discussion to the roles of tRNAs in translation. In mammalian cells, tRNAs regulate many processes, either as intact molecules or fragments (reviewed in Berg and Brandl 2021). It is possible that mistranslating tRNA variants affect biological processes in a translation independent manner.

\section{Mistranslating alanine at proline codons with tRNA Gro $_{\text {P70 }}$}

We first identified alleles encoding tRNA $\mathrm{G}_{\mathrm{G} 3: \mathrm{U70}}^{\mathrm{P} 0}$ (Pro $\rightarrow$ Ala) as spontaneous suppressors of a conditional tti2-L187P allele (Hoffman et al. 2017). The suppressor tRNA inserts alanine at the proline codon at position 187 in tti2, which restores nearly full function to Tti2. Mistranslation is due to a single base change of C70 to $\mathrm{T}$ in a tRNA $\mathrm{UGG}_{\mathrm{UG}}^{\text {Pro }}$ encoding gene. In the tti2-based selection, we identified four independent tRNA $\mathrm{UGG}_{\mathrm{UG}}^{\text {Pro }}$ genes with this mutation and estimate that it occurs at a frequency of approximately $10^{-6}$ in yeast populations. tRNAs with G3: C70 base pairs, a single mutation removed from creating the major G3: U70 alanine identity element, are found in bacteria, archaea, and yeast species, but are rare in eukaryotes other than yeast (Berg et al. 2018). tRNA Lys:U70 variants have been isolated from E. coli (see Prather et al. 1984). Combined with the prevalence of $\mathrm{tRNA}_{\mathrm{G} 3: U 70}^{\text {Pro }}$ in yeast populations, these findings suggest that in single cell organisms the ability to mistranslate codons as alanine may provide an advantage to the population. The absence of G3: C70 in most eukaryotic tRNAs suggests that the mistranslation resulting from a change to U70 would be deleterious for these species.

In summary, mistranslating tRNAs impact cells in ways that are specific to the type of mistranslation. The consequences of these mistranslating tRNAs will in turn depend upon the genotype of the organism and the environment. It will be important to consider these differences when analyzing tRNAs as genetic modifiers of disease.

\section{Data availability}

Strains and plasmids are available upon request. The authors affirm that all data necessary for confirming the conclusions of the article are present within the article, figures, and supplementary material. Supplementary files are available at figshare: https:// doi.org/10.25387/g3.14580009. Supplementary File S1 contains all supplementary tables and figures. Supplementary File S2 contains raw and validated SGA data. Supplementary File S3 contains $\mathrm{R}$ code used to analyze mass spectrometry and RNA sequencing count data. The gene expression data from the RNA sequencing analysis have been deposited in NCBI's Gene Expression Omnibus (Edgar et al. 2002) and are accessible through GEO Series accession number GSE174145. The mass spectrometry proteomics data have been deposited to the ProteomeXchange Consortium via the PRIDE (Perez-Riverol et al. 2019) partner repository with the dataset identifier PXD025934. 


\section{Acknowledgments}

The authors would like to thank Ricard Rodriguez-Mias for assisting with the mass spectrometry and maintaining the instruments, Mario Leutert for assistance with the R2P1 sample preparation and data collection, Stephanie Zimmerman for providing the code to analyze codon distribution in mistranslated peptides, Onn Brandman for the HSE-GFP reporter plasmid, Brenda Andrews for yeast strain Y7092 and Ecaterina Cozma for critical reading of the manuscript.

\section{Funding}

This work was supported from the Natural Sciences and Engineering Research Council of Canada (NSERC; RGPIN-201504394 to C.J.B.), the Canadian Institutes of Health Research (FDN159913 to G.W.B.) and generous donations from Graham Wright and James Robertson to M.D.B. Mass spectrometry work was supported by a research grant from the Keck Foundation, NIH grant R35 GM119536 and associated instrumentation supplement (to J.V.). M.D.B. was supported by an NSERC Alexander Graham Bell Canada Graduate Scholarship (CGS-D). J.I. holds an NSERC Alexander Graham Bell Canada Graduate Scholarship (PGS-D). B.Y.R. holds an Natural Science Foundation Graduate Research Fellowship (DGE-1256082).

\section{Conflicts of interest}

None declared.

\section{Literature cited}

Asahara H, Himeno H, Tamura K, Nameki N, Hasegawa T, et al. 1994. Escherichia coli seryl-tRNA synthetase recognizes tRNA ${ }^{\text {Ser }}$ by its characteristics tertiary structure. J Mol Biol. 236:738-748. doi: 10.1006/jmbi.1994.1186.

Ben-Aroya S, Coombes C, Kwok T, O'Donnell KA, Boeke JD, et al. 2008. Toward a comprehensive temperature-sensitive mutant repository of the essential genes of Saccharomyces cerevisiae. Mol Cell. 30: 248-258. doi:10.1016/j.molcel.2008.02.021.

Benzer S, Champe SP. 1962. A change from nonsense to sense in the genetic code. Proc Natl Acad Sci USA. 48:1114-1121. doi: 10.1073/pnas.48.7.1114.

Berg MD, Zhu Y, Isaacson J, Genereaux J, Loll-Krippleber R, et al.. 2020. Chemical-Genetic Interactions with the Proline Analog L-Azetidine-2-Carboxylic Acid in Saccharomyces cerevisiae. G3 (Bethesda). 10:4335-4345.

Berg MD, Brandl CJ. 2021. Transfer RNAs: diversity in form and function. RNA Biol. 18:316-339.

Berg MD, Genereaux J, Zhu Y, Mian S, Gloor GB, et al. 2018. Acceptor stem differences contribute to species-specific use of yeast and human tRNA ${ }^{\text {Ser }}$. Genes (Basel). 9:612.

Berg MD, Giguere DJ, Dron JS, Lant JT, Genereaux J, et al. 2019a. Targeted sequencing reveals expanded genetic diversity of human transfer RNAs. RNA Biol. 16:1574-1585.

Berg MD, Hoffman KS, Genereaux J, Mian S, Trussler RS, et al. 2017. Evolving mistranslating tRNAs through a phenotypically ambivalent intermediate in Saccharomyces cerevisiae. Genetics. 206: 1865-1879. doi:10.1534/genetics.117.203232.

Berg MD, Zhu Y, Genereaux J, Ruiz BY, Rodriguez-Mias RA, et al. 2019b. Modulating mistranslation potential of $\mathrm{tRNA}^{\mathrm{Ser}}$ in Saccharomyces cerevisiae. Genetics. 213:849-863. doi:10.1534/genetics.119.302525.
Biou V, Yaremchuk A, Tukalo M, Cusack S. 1994. The 2.9 A crystal structure of $\mathrm{T}$. thermophilus seryl-tRNA synthetase complexed with tRNA ${ }^{\text {Ser }}$. Science. 263:1404-1410. doi:10.1126/science. 8128220.

Bolger AM, Lohse M, Usadel B. 2014. Trimmomatic: a flexible trimmer for Illumina sequence data. Bioinformatics. 30:2114-2120. doi:10.1093/bioinformatics/btu170.

Brachmann CB, Davies A, Cost GJ, Caputo E, Li J, et al. 1998. Designer deletion strains derived from Saccharomyces cerevisiae S288C: a useful set of strains and plasmids for PCR-mediated gene disruption and other applications. Yeast. 14:115-132. doi:10.1002/ (SICI)1097-0061(19980130)14:2<115::AID-YEA204>3.0.CO;2-2.

Brandman O, Stewart-Ornstein J, Wong D, Larson A, Williams CC, et al. 2012. A ribosome-bound quality control complex triggers degradation of nascent peptides and signals translation stress. Cell. 151:1042-1054. doi:10.1016/j.cell.2012.10.044.

Breslow DK, Cameron DM, Collins SR, Schuldiner M, StewartOrnstein J, et al. 2008. A comprehensive strategy enabling high-resolution functional analysis of the yeast genome. Nat Methods. 5:711-718. doi:10.1038/nmeth.1234.

Carbon J, Berg P, Yanofsky C. 1966. Studies of missense suppression of the tryptophan synthetase A-protein mutant A36. Proc Natl Acad Sci USA. 56:764-771. doi:10.1073/pnas.56.2.764.

Chapeville F, Lipmann F, Ehrenstein GV, Weisblum B, Ray WJ, et al. 1962. On the role of soluble ribonucleic acid in coding for amino acids. Proc Natl Acad Sci USA. 48:1086-1092. doi:10.1073/pnas. 48.6.1086.

Costanzo M, VanderSluis B, Koch EN, Baryshnikova A, Pons C, et al. 2016. A global genetic interaction network maps a wiring diagram of cellular function. Science. 353:aaf1420-14. doi:10.1126/science.aaf1420

Crawford IP, Yanofsky C. 1959. The formation of a new enzymatically active protein as a result of suppression. Proc Natl Acad Sci USA. 45:1280-1287. doi:10.1073/pnas.45.8.1280.

Dayhoff MG, Eck RV. 1968. Atlas of Protein Sequence and Structure. Silver Spring, MD; Natd Biomed Res Found

Dobin A, Davis CA, Schlesinger F, Drenkow J, Zaleski C, et al. 2013. STAR: ultrafast universal RNA-seq aligner. Bioinformatics. 29: 15-21. doi:10.1093/bioinformatics/bts635.

de Duve C. 1988. Transfer RNAs: the second genetic code. Nature. 333:117-118. doi:10.1038/333117a0.

Edgar R, Domrachev M, Lash AE. 2002. Gene expression omnibus: NCBI gene expression and hybridization array data repository. Nucleic Acids Res. 30:207-210. doi:10.1093/nar/30.1.207.

Eng JK, Jahan TA, Hoopmann MR. 2013. Comet: an open-source MS/MS sequence database search tool. Proteomics. 13:22-24. doi: 10.1002/pmic.201200439.

Engel SR, Dietrich FS, Fisk DG, Binkley G, Balakrishnan R, et al. 2014. The reference genome sequence of Saccharomyces cerevisiae: then and now. G3 (Bethesda). 4:389-398. doi:10.1534/g3.113.008995.

Francklyn C, Schimmel P. 1989. Aminoacylation of RNA minihelices with alanine. Nature. 337:478-481.

Geslain R, Cubells L, Bori-Sanz T, Álvarez-Medina R, Rossell D, et al. 2010. Chimeric tRNAs as tools to induce proteome damage and identify components of stress responses. Nucleic Acids Res. 38: e30. doi:10.1093/nar/gkp1083.

Giegé R, Sissler M, Florentz C. 1998. Universal rules and idiosyncratic features in tRNA identity. Nucleic Acids Res. 26:5017-5035. doi: 10.1093/nar/26.22.5017.

Goodman HM, Olson MV, Hall BD. 1977. Nucleotide sequence of a mutant eukaryotic gene: the yeast tyrosine-inserting ochre suppressor SUP4-0. Proc Natl Acad Sci USA. 74:5453-5457. doi: 10.1073/pnas.74.12.5453. 
Gorini L, Beckwith JR. 1966. Suppression. Annu Rev Microbiol. 20: 401-422. doi:10.1146/annurev.mi.20.100166.002153.

Gupta NK, Khorana HG. 1966. Missense suppression of the tryptophan synthetase A-protein mutant A78. Proc Natl Acad Sci USA. 56:772-779. doi:10.1073/pnas.56.2.772.

Henikoff S, Henikoff JG. 2000. Amino acid substitution matrices. Adv Protein Chem. 54:73-97. doi:10.1016/s0065-3233(00)54003-0.

Himeno H, Yoshida S, Soma A, Nishikawa K. 1997. Only one nucleotide insertion to the long variable arm confers an efficient serine acceptor activity upon Saccharomyces cerevisiae tRNA ${ }^{\text {Leu }}$ in vitro. J Mol Biol. 268:704-711. doi:10.1006/jmbi.1997.0991.

Hirsh D. 1971. Tryptophan transfer RNA as the UGA suppressor. J Mol Biol. 58:439-458. doi:10.1016/0022-2836(71)90362-7.

Hoffman KS, Berg MD, Shilton BH, Brandl CJ, O’Donoghue P. 2017a. Genetic selection for mistranslation rescues a defective co-chaperone in yeast. Nucleic Acids Res. 45:3407-3421. doi: 10.1093/nar/gkw1021.

Hoffman KS, O’Donoghue P, Brandl CJ. 2017b. Mistranslation: from adaptations to applications. Biochim Biophys Acta. 1861: 3070-3080. doi:10.1016/j.bbagen.2017.01.031.

Hottiger T, Schmutz P, Wiemken A. 1987. Heat-induced accumulation and futile cycling of trehalose in Saccharomyces cerevisiae. J Bacteriol. 169:5518-5522. doi:10.1128/jb.169.12.5518-5522.1987.

Hou YM, Schimmel P. 1988. A simple structural feature is a major determinant of the identity of a transfer RNA. Nature. 333:140-145. doi:10.1038/333140a0.

Hou YM, Schimmel P. 1989. Evidence that a major determinant for the identity of a transfer RNA is conserved in evolution. Biochemistry. 28:6800-6804. doi:10.1021/bi00443a003.

Imura N, Weiss GB, Chambers RW. 1969. Reconstitution of alanine acceptor activity from fragments of yeast tRNA Ala . Nature. 222: 1147-1148. doi:10.1038/2221147a0.

Jones DS, Nishimura S, Khorana HG. 1966. Studies on polynucleotides: LVI. Further syntheses, in vitro, of copolypeptides containing two amino acids in alternating sequence dependent upon DNA-like polymers containing two nucleotides in alternating sequence. J Mol Biol. 16:454-472. doi:10.1016/S0022-2836(66) 80185-7.

Jongkamonwiwat N, Ramirez MA, Edassery S, Wong ACY, Yu J, et al. 2020. Noise exposures causing hearing loss generate proteotoxic stress and activate the proteostasis network. Cell Rep. 33: 108431.doi:10.1016/j.celrep.2020.108431.

Joshi K, Cao L, Farabaugh PJ. 2019. The problem of genetic code misreading during protein synthesis. Yeast. 36:35-42. doi: 10.1002/yea.3374.

Käll L, Canterbury JD, Weston J, Noble WS, MacCoss MJ. 2007. Semi-supervised learning for peptide identification from shotgun proteomics datasets. Nat Methods. 4:923-925. doi:10.1038/ nmeth1113.

Kofoed M, Milbury KL, Chiang JH, Sinha S, Ben-Aroya S, et al. 2015. An updated collection of sequence barcoded temperature-sensitive alleles of yeast essential genes. G3 (Bethesda). 5:1879-1887. doi: 10.1534/g3.115.019174

Koonin EV, Novozhilov AS. 2017. Origin and evolution of the universal genetic code. Annu Rev Genet. 51:45-62. doi:10.1146/annurev-genet-120116-024713.

Labbadia J, Morimoto RI. 2015. The biology of proteostasis in aging and disease. Annu Rev Biochem. 84:435-464. doi:10.1146/annurev-biochem-060614-033955.

Lant JT, Berg MD, Sze DH, Hoffman KS, Akinpelu IC, et al. 2018. Visualizing tRNA-dependent mistranslation in human cells. RNA Biol. 15:567-575. doi:10.1080/15476286.2017.1379645.
Lant JT, Berg MD, Heinemann IU, Brandl CJ, O’Donoghue P. 2019. Pathways to disease from natural variations in human cytoplasmic tRNAs. J Biol Chem. 294:5294-5308. doi:10.1074/jbc.rev118. 002982.

Lee JW, Beebe K, Nangle LA, Jang J, Longo-Guess CM, et al. 2006. Editing-defective tRNA synthetase causes protein misfolding and neurodegeneration. Nature. 443:50-55. doi:10.1038/nature05096.

Leutert M, Rodríguez-Mias RA, Fukuda NK, Villén J. 2019. R2-P2 rapid-robotic phosphoproteomics enables multidimensional cell signaling studies. Mol Syst Biol. 15:1-20. doi:10.15252/msb. 20199021.

Li Z, Vizeacoumar FJ, Bahr S, Li J, Warringer J, et al. 2011. Systematic exploration of essential yeast gene function with temperature-sensitive mutants. Nat Biotechnol. 29:361-367. doi: 10.1038/nbt.1832.

Liao Y, Smyth GK, Shi W. 2014. FeatureCounts: an efficient general purpose program for assigning sequence reads to genomic features. Bioinformatics. 30:923-930. doi:10.1093/bioinformatics/btt656.

Lillie SH, Pringle JR. 1980. Reserve carbohydrate metabolism in Saccharomyces cerevisiae: responses to nutrient limitation. J Bacteriol. 143:1384-1394. doi:10.1128/jb.143.3.1384-1394.1980.

Liu Y, Satz JS, Vo M-N, Nangle LA, Schimmel P, et al. 2014. Deficiencies in tRNA synthetase editing activity cause cardioproteinopathy. Proc Natl Acad Sci USA. 111:17570-17575. doi: 10.1073/pnas.1420196111.

Love MI, Huber W, Anders S. 2014. Moderated estimation of fold change and dispersion for RNA-seq data with DESeq2. Genome Biol. 15:550-521. doi:10.1186/s13059-014-0550-8.

Loveland AB, Demo G, Grigorieff N, Korostelev AA. 2017. Ensemble cryo-EM elucidates the mechanism of translation fidelity. Nature. 546:113-117. doi:10.1038/nature22397.

Lu J, Bergert M, Walther A, Suter B. 2014. Double-sieving-defective aminoacyl-tRNA synthetase causes protein mistranslation and affects cellular physiology and development. Nat Commun. 5: 5650.doi:10.1038/ncomms6650.

McClain WH, Foss K. 1988. Changing the identity of a tRNA by introducing a G-U wobble pair near the 3' acceptor end. Science. 240: 793-796.

McLendon PM, Robbins J. 2015. Proteotoxicity and cardiac dysfunction. Circ Res. 116:1863-1882. doi:10.1161/CIRCRESAHA.116. 305372.

Mohler K, Ibba M. 2017. Translational fidelity and mistranslation in the cellular response to stress. Nat Microbiol. 2:17117. doi: 10.1038/nmicrobiol.2017.117.

Navarrete-Perea J, Gygi SP, Paulo JA. 2021. Growth media selection alters the proteome profiles of three model microorganisms. J Proteomics. 231:104006.doi:10.1016/j.jprot.2020.104006.

Ogle JM, Murphy IV FV, Tarry MJ, Ramakrishnan V. 2002. Selection of tRNA by the ribosome requires a transition from an open to a closed form. Cell. 111:721-732. doi:10.1016/S0092-8674(02) 01086-3.

Parrou JL, Teste MA, François J. 1997. Effects of various types of stress on the metabolism of reserve carbohydrates in Saccharomyces cerevisiae: genetic evidence for a stress-induced recycling of glycogen and trehalose. Microbiology. 143:1891-1900. doi:10.1099/002212 87-143-6-1891

Perez-Riverol Y, Csordas A, Bai J, Bernal-Llinares M, Hewapathirana S, et al. 2019. The PRIDE database and related tools and resources in 2019: improving support for quantification data. Nucleic Acids Res. 47:D442-D450. doi:10.1093/nar/gky1106.

Prather NE, Murgola EJ, Mims BH. 1984. Nucleotide substitution in the amino acid acceptor stem of lysine transfer RNA causes 
missense suppression. J Mol Biol. 172:177-184. doi:10.1016/S00222836(84)80036-4.

Rich A, RajBhandary UL. 1976. Transfer RNA: molecular structure, sequence, and properties. Annu Rev Biochem. 45:805-860. doi: 10.1146/annurev.bi.45.070176.004105.

Ruan B, Palioura S, Sabina J, Marvin-Guy L, Kochhar S, et al. 2008. Quality control despite mistranslation caused by an ambiguous genetic code. Proc Natl Acad Sci USA. 105:16502-16507. doi: 10.1073/pnas.0809179105

Santos M, Pereira PM, Varanda AS, Carvalho J, Azevedo M, et al. 2018. Codon misreading tRNAs promote tumor growth in mice. RNA Biol. 15:773-786. doi:10.1080/15476286.2018.1454244.

Schneider CA, Rasband WS, Eliceiri KW. 2012. NIH Image to ImageJ: 25 years of image analysis. Nat Methods. 9:671-675. doi: 10.1038/nmeth.2089

Shannon P, Markiel A, Ozier O, Baliga NS, Wang JT, et al. 2003. Cytoscape: a software environment for integrated models of biomolecular interaction networks. Genome Res. 13:2498-2504. doi: 10.1101/gr.1239303

Shcherbakov D, Teo Y, Boukari H, et al. 2019. Ribosomal mistranslation leads to silencing of the unfolded protein response and increased mitochondrial biogenesis. Commun Biol. 2:381.

Singer MA, Lindquist S. 1998. Multiple effects of trehalose on protein folding in vitro and in vivo. Mol Cell. 1:639-648. doi: 10.1016/S1097-2765(00)80064-7.

Sprouffske K, Wagner A. 2016. Growthcurver: an R package for obtaining interpretable metrics from microbial growth curves. BMC Bioinformatics. 17:17-20. doi:10.1186/s12859-016-1016-7.

Stadler J, Yanofsky C. 1959. Studies on a series of tryptophanindependent strains derived from a tryptophan-requiring mutant of Escherichia coli. Genetics. 44:105-123.
Supek F, Bošnjak M, Škunca N, Šmuc T. 2011. Revigo summarizes and visualizes long lists of gene ontology terms. PLoS One. 6: e21800. 6: doi:10.1371/journal.pone.0021800.

Tong AH, Evangelista M, Parsons AB, Xu H, Bader GD, et al. 2001 Systematic genetic analysis with ordered arrays of yeast deletion mutants. Science. 294:2364-2368. doi:10.1126/science.1065810.

Wagih O, Usaj M, Baryshnikova A, VanderSluis B, Kuzmin E, et al. 2013. SGAtools: one-stop analysis and visualization of array-based genetic interaction screens. Nucleic Acids Res. 41: 591-596. doi:10.1109/ICCSE.2016.7581644.

Willis MS, Patterson C. 2013. Proteotoxicity and cardiac dysfunction - Alzheimer's disease of the heart?. N Engl J Med. 368:455-464 doi:10.1056/nejmra1106180.

Woese CR, Dugre DH, Saxinger WC, Dugre SA. 1966. The molecular basis for the genetic code. Proc Natl Acad Sci USA. 55:966-974. doi:10.1073/pnas.55.4.966.

Yanofsky C, Crawford IP. 1959. The effects of deletions, point mutations, reversion and suppressor mutations on the two components of the tryptophan synthetase of Escherichia coli. Proc Natl Acad Sci USA. 45:1016-1026. doi:10.1073/pnas.45.7.1016.

Zhu Y, Berg MD, Yang P, Loll-Krippleber R, Brown GW, et al. 2020. Mistranslating tRNA identifies a deleterious S213P mutation in the Saccharomyces cerevisiae eco1-1 allele. Biochem Cell Biol. 98: 624-630. doi:10.1139/bcb-2020-0151.

Zimmerman SM, Kon Y, Hauke AC, Ruiz BY, Fields S, et al. 2018 Conditional accumulation of toxic tRNAs to cause amino acid misincorporation. Nucleic Acids Res. 46:7831-7843. doi:10.1093/ nar/gky623.

Communicating editor: C. S. Hoffman 